\title{
Reviewer Thank You
}

Published online: 5 January 2013

(C) American College of Medical Toxicology 2012

The Editorial staff of the Journal of Medical Toxicology appreciates all of those who make the success of the journal possible. A medical journal cannot exist without the tireless, and often thankless, work of its reviewers. The list only includes those reviewers registered on our web-based program, Editorial Manager, so many who have reviewed may not be on the list. To those reviewers omitted, we apologize, but also thank you very much.

Cynthia Aaron

Mohamed S Abdel-Rahman

Mohammad Abdollahi

Redouane Abouqal

Terry Adirim

Ritesh Agarwal

Steven E Aks

Walter Aquiles Alarcon

Timothy Albertson

D Adam Algren*

Thomas Arnold

Gavin Arteel

Kavita M Babu

Murl Bailey

Cornelia Baines

Salome Ballesteros

Lydia Baltarowich

Theodore Bania

William Banner

Donald Barceloux

Carola Bardage

Dana Boyd Barr

Gillian Barratt
Fermin Barrueto

J Dave Barry

D Nicholas Bateman

Stuart Batterman

Frederic Baud

Martin Belson

John G Benitez

Jeffrey Bernstein

Phillippe J Berny

Michael C Beuhler

Steven B Bird*

Kenneth Bizovik

Dennis Blodgett

G Randall Bond

Jonathan Borak

Stephen W Borron

Mehmet Bosnak

George Bosse

Edward W Boyer

Leslie Boyer

Eva Boyez

Gokcen Bozdag

Sally Bradberry

Geroge Braitberg
Jeffrey Brent*

Stephane Bretagne

Daniel E Brooks*

David Eric Brush

Ahna G Brutlag

Sean M Bryant*

Jennie Alison Buchanan

Alan Buchwald

Nick Buckley

Jeff Burgess

Keith Burkhart

Howard Burns

Michael Burns

Michele Burns Ewald

Sean Bush

Diane P Calello

Lee Cantrell

Martin Caravati

Shaun D Carstairs

Marcel Casavant

Grant Cave

Edward Cetaruk

Gar Ming Chan 
Arthur S Chang

Nathan P Charlton

Lu Chensheng

Roger Chou

Michael Ray Christian

Alvin F Chu

Ryan Chuang

Richard James Church

Peter Chyka

John Cienki

James Cisek

Cathleen Clancy

Rick Clark

Aaron Collins

James Courtney

Robert D Cox

Barbara I Crouch

Kirk Lawrence Cumpston

Steven Curry

John Curtis

John Ashton Curtis

Paul Dargan

Richard C Dart

Cedric Daubin

Andrew Dawson

Arthur de Meijer

HJ De Silva

Kathleen Delaney

Joao Delgado

Mary Derby

Francis DeRoos

Christoer R DeWitt

James Henry Diaz

Jose Diaz-Alcala

Ricardo Dinis-Oliveira

Robert Paulwsett

Eric Dunayer

Bertrand Dupont

Francois Durand

Leslie Dye

Soia Dyer

Michael Eddleston

David Eldridge

Kristin Engebretsen

Jennifer Englund

Tim Evans

Brenna M Farmer

Mariya Faheem Farooqi

Miguel Fernandez
Jeffrey Fine

Mathias B Forrester

Jessica Fulton

Brent Furbee

David Gaist

Michael Ganetsky

Martin Ganzert

R Garcia

Concetta Gardi

Tam Garland

Cynthia Gaskill

Marc Gautreau

Ann-Jeannette Geib

Richard Geller

Robert Jerome Geller

Mathew George

Richard Gerkim

Hassan Ghasem

Beth Ginsburg

Barry Gold

David S Goldfarb

Daniel A Goldstein

Christoer Gordon

Ronald Gots

Kimberlie Graeme

John Graham

Andis Graudins

Michael Greenberg

Howard A Greller

David Gummin

Naren Gunja

Ramesh C Gupta

Leon Gussow

Geeta Gyamlani

Jason Hack

In-Hei Hahn

Alan H Hall

Jeffery Owen Hall

Christine Haller

Jennifer Hannum

Fred Harchelroad

Lewis S Hardison Jr

Martyn Harvey

Bryan Hayes

Kennon Heard*

Matthew Hedge

Alan C Heffner

Robert Hendrickson

Fred Henretig

David J Hewitt
James Hillman

Michael Hodgman*

Ernest Hodgson

Robert J Hoffman

Robert Steven Hoffman

Jonas Hojer

Joel Holger

Michael Holland*

Christoer Holstege

William Holubek

Jason Hoppe

Zane Horowitz

Knut Erik Hovda

Mary Ann Howland

Oliver Hung

Katie Hurlbut

Danyal Ibrahim

Geoffrey Isbister

Kelly Johnson-Arbor

Heath Jolliff

James Hughes Jones

Jerry Jones

Prashant Joshi

Bryan S Judge

Tamar Kadar

Louise Kao

John Kashani

Kenneth Katz

Ziad N Kazzi

Kai Kehe

Anne-Maree Kelly

Catherine Kelly

Carlton Kempter

William Kerns II*

D Chris Keyes

Mohamed Abdel-Azeem Khalaf

Eun-Joo Kim

Paul G King

Mark Kirk

Barbara Kirrane

Ron Kirschner

Craig Sims Kitchens

Kurt C Kleinschmidt

OR Koch

Aleš Kogoj

Michael Kosnett

Mark Kostic

Edward Krenzelok

Allyson A Kreshak 
Ed Kuffner

Ken Kulig

Grant Lackey

Melisa Lai

Rafael Laniado-Laborı'n

Patrick McCafferty Lank

Eric J Lavonas

Chris Le

Jerrold B Leikin

Vivian Leung

Michael Levine*

Howard Levitin

Pilippe Lheureux

Erica Liebelt

Ja-Liang Lin

Toby Litovitz

Heather Long

Sara Monica Long

J D Lorenc

Jeffrey Love

Frank Lovecchio

Debbie L LoVerde, Jr.

Jennifer Lowry

Jenny J Lu

Po-Yung Lu

Tsung-Hsueh Lu

Andres Lugo

Binh Ly

James Malcolm Madsen

Gerald Maloney

Alex F Manini

Mary-Elise Manuell

Steven Matthew Marcus

Kathy Marquardt

Zarko Martinovic

Michael Matteucci

Maryann Mazer-Amirshahi

Fred McDonald

Charles Alan McKay

Nathanael J McKeown

Timothy J Meehan

Bruno Megarbane

William Joel Meggs

M John Mendelsohn

Melissa A Miller

Michael Miller

Kirk Mills

Alicia Minns

David L Morgan

Michelle Mostrom
Allan R Mottram

Rita Mrvos

Michael Mullins*

Steen Munday

David Munter

Christine M Mury

Lisa Mury

Mike Mury

Mark B Mycyk*

T Narahashi

Ana Navas-Acien

Jamie L Nelsen

Lewis Nelson

Mike Nelson

KA Neville

Adeline Su-Yin Ngo

P Nisse

Patrice Nodet

Josh Nogar

Sean Nordt

Semir Nouira

Jennifer Oakes

Ayrn O'Connor

Fred Oehme

Dean Olsen

Kent Olson

Gerald Francis O'Malley*

Thomas Osimitz

Kai Osterberg

Kevin Osterhoudt

John Osterloh

Mary Palmer

Robert Palmer

Frank Paloucek

Manish Patel

Len Paulozzi

Julie Pavlin

Tamas Peredy

Jeanmarie Perrone

Scott illips

Jessica Pierog

Steven Pike

Anthony F Pizon

R Ponampalam

Robert Poppenga

Laura Elizabeth Power

Laurie Prescott

Jane Prosser

Alex Proudfoot
Roy Purssell

Lawrence Quang

Rajendra Raizada

Fatima Ramon

Davud Ray

Michael Reed

Arne Reimers

Jose Rella

Sean Hyun Rhyee

William Richardson

Brad Riley

Raymond Roberge

Renee Robinson

Gary Rockwood

MG Roma

Rutherfoord Rose

Patricia Rosen

Christoer D Rosenbaum

Jonathan Rosenson

Anna Rouse Dulaney

Annalisa Rubino

Anne-Michelle Ruha

Wilson Rumbeiha

Daniel E Rusyniak

Eva Saedder

Steven Salhanick

Matthew Salzman

Anthony Scalzo

Tammi Schaeffer

Elizabeth Scharman

Jay Schauben

Joshua G Schier

James Schmidt

Aaron Schneir

Heather Schumann

David Schwartz

Michael Schwartz

Donna Seger

Gilad Segev

Robert Seidner

Steven A Seifert

Genna Sellers

Shahin Shadnia

Beth Shortridge

S Singh

Marco LA Sivilottic

Carl G Skinner

Silas W Smith

William Smith 
Susan Smolinske

Curtis P Snook*

Henry Alfred Spiller

Frazier Stevenson

Andrew Stolbach

Christine M Stork

Mark Su*

Jeffrey Suchard*

Daniel Sudakin

John Burke Sullivan

Patricia Talcott

David Tanen

Asim F Tarabar

Daniel Thau Teitelbaum

Brigham Temple

Wayne Temple

Milton Tenenbein

Jerry Thomas

Simon Thomas

Trevonne Thompson

Josef G Thundiyil

John Tilelli
Heather Tiller

Karine Titier

Anthony Tomassoni

Christian A Tomaszewski

Rebecca Tominack

Theodore Tong

Richard T Tovar

Steen Traub

Lauren Trilli

Geoffrey T Tucker

Matthew Valento

Susi Vassallo

ND Vazini

David Vearrier

Larissa I Velez

Christine Vogeli

Rais Vohra

Javier Waksman

Frank Walter

Richard Wang

Jeanine Ward
William Steen Waring

Paul M Wax

Ashley Webbc

Patrick L West

Kenneth S Whitlow

Ian Macgregor Whyte

Timothy J Wiegand*

Brandon Wills

Chris Winder

Mary Wittler

Amy Wolkin

David M Wood

Alan Woolf

Robert Wright

Mark Yarema

Luke Yip

Michael Young

Eduard Zaloshnja

Michele Zell-Kanter

Christina Zhang

Deborah Zvosec

*Reviewers who have completed more than ten reviews since the institution of Editorial Manager. 